\title{
Ewing Sarcoma in the Sciatic Nerve: Case Report
}

\section{Sarcoma de Ewing no nervo ciático: relato de caso}

\author{
Robinson Antonio Menegotto Marques ${ }^{10}$ Adriana Hernandez Marques ${ }^{2(10}$ \\ Amanda Hernandez Marques 3 (1) \\ ${ }^{1}$ Department of Neurosurgery, Hospital Vita Curitiba, Curitiba, PR, \\ Brazil \\ 2 Medical School, Universidade Federal do Paraná (UFPR), Curitiba, \\ Brazil \\ ${ }^{3}$ Medical School, Pontifícia Universidade Católica do Paraná (PUCPR), \\ Curitiba, Brazil \\ Arq Bras Neurocir

\begin{abstract}
Address for correspondence Robinson Antonio Menegotto Marques, MD, Departmento de Neurocirurgia, Hospital Vita Curitiba, Curitiba, PR, Brazil (e-mail: menegottom@yahoo.com.br).
\end{abstract}

\section{Introduction}

Ewing sarcoma (ES) is an uncommon malignant neoplasm that most often affects the bones of children and adoles- cents. ${ }^{1}$ In $\sim 25 \%$ of the cases, ES can originate in soft tissues. ${ }^{2}$ Currently, these tumors are included in the Ewing Sarcoma Family of Tumors (ESFT), because they have the same histological and genotypic characteristics. ${ }^{3}$ Extraosseous ES received

April 15, 2021

accepted

June 16, 2021
DOI https://doi.org/

10.1055/s-0041-1739271. ISSN 0103-5355.

\footnotetext{
(c) 2022. Sociedade Brasileira de Neurocirurgia. All rights reserved. This is an open access article published by Thieme under the terms of the Creative Commons Attribution-NonDerivative-NonCommercial-License, permitting copying and reproduction so long as the original work is given appropriate credit. Contents may not be used for commercial purposes, or adapted, remixed, transformed or built upon. (https://creativecommons.org/ licenses/by-nc-nd/4.0/)

Thieme Revinter Publicações Ltda., Rua do Matoso 170, Rio de Janeiro, RJ, CEP 20270-135, Brazil
} 
originating from peripheral nerves is very rare. We present a case of extraskeletal ES originating in a young patient's sciatic nerve, and discuss the great challenge of treating patients with this pathology.

\section{Case Report}

A 27-year-old, Caucasian, male patient presented with complaints of progressive loss of strength in the left foot with 13 months of evolution associated with pain in the back of the left thigh with proximal and distal irradiation. Upon examination, the patient was in good general condition, with café-au-lait patches on the abdominal wall, without other stigmata of type-1 neurofibromatosis. He had a palpable mass between the middle and lower thirds of the left posterior thigh, of $\sim 5 \mathrm{~cm}$, mobile, non-pulsatile, painful, and with a positive Tinel sign. Upon auscultation, no murmurs were perceived. The neurological examination showed grade-0 strength for hallux extension and dorsiflexion, and grade- 1 strength for left foot eversion, and tactile and painful hypoesthesia on the side of the left leg and foot. An MRI scan identified an oval, circumscribed tumor in the left sciatic nerve, without affecting the neighboring planes, without signs of edema surrounding it, with annular uptake of contrast suggestive of schwannoma/neurofibroma (-Fig. 1). An electroneuromyography exam identified axonal involvement of the left sciatic/common fibular nerve, with signs of denervation in muscles innervated by the left fibular nerve. Surgical treatment was applied, with access to the back of the left thigh to expose the sciatic nerve and the origin of the left tibial and common fibular nerves. A tumor of $\sim 7 \mathrm{~cm}$ in length and $3.2 \mathrm{~cm}$ in its largest diameter was identified, located in the final and lateral portions of the sciatic nerve and in the origin of the common fibular nerve (-Fig. 2). Circumferential dissection was performed, and no adherence to neighboring tissues was observed. The tumor was easily detached from the tibial nerve. The fascicle where the tumor originated was identified in the final portion of the sciatic nerve. Electrical stimulation showed no response. The tumor was resected en bloc, sparing a fascicle of the common fibular nerve. The sectioned fascicle was reconstructed with two $8-\mathrm{cm}$ sural nerve cables each. A pathology examination described a malignant neoplasm of small cells of high cellularity and with areas of necrosis. The immunohistochemical study was negative for antibodies CKAE1/AE3, S100, actin, desmin, CK7, synaptophysin and positive for CD 99, CD 56, vimentin, BcL-2, and rarely positive in neoplastic cells for EMA. A total of $70 \%$ of the neoplastic cells were positive for Ki67. (-Fig. 3). These characteristics were compatible with the diagnosis of peripheral primitive neuroectodermal tumor (PNET)/Ewing sarcoma. Clinical staging was performed with chest tomography, whole-body scintigraphy and bone marrow biopsy, without evidence of metastatic tumor. Chemotherapy started with vincristine, doxorubicin, and cyclophosphamide (VDC) alternated with ifosfamide and etoposide (IE) every two weeks (the VDC-IE combination) for a year of treatment, complemented with local radiotherapy. The treatment was well tolerated by the patient, who evolved with improved sensitivity in the left leg and foot, improved strength in dorsiflexion of the foot (M3) and non-functional improvement in hallux extension and eversion of the foot (M1). The
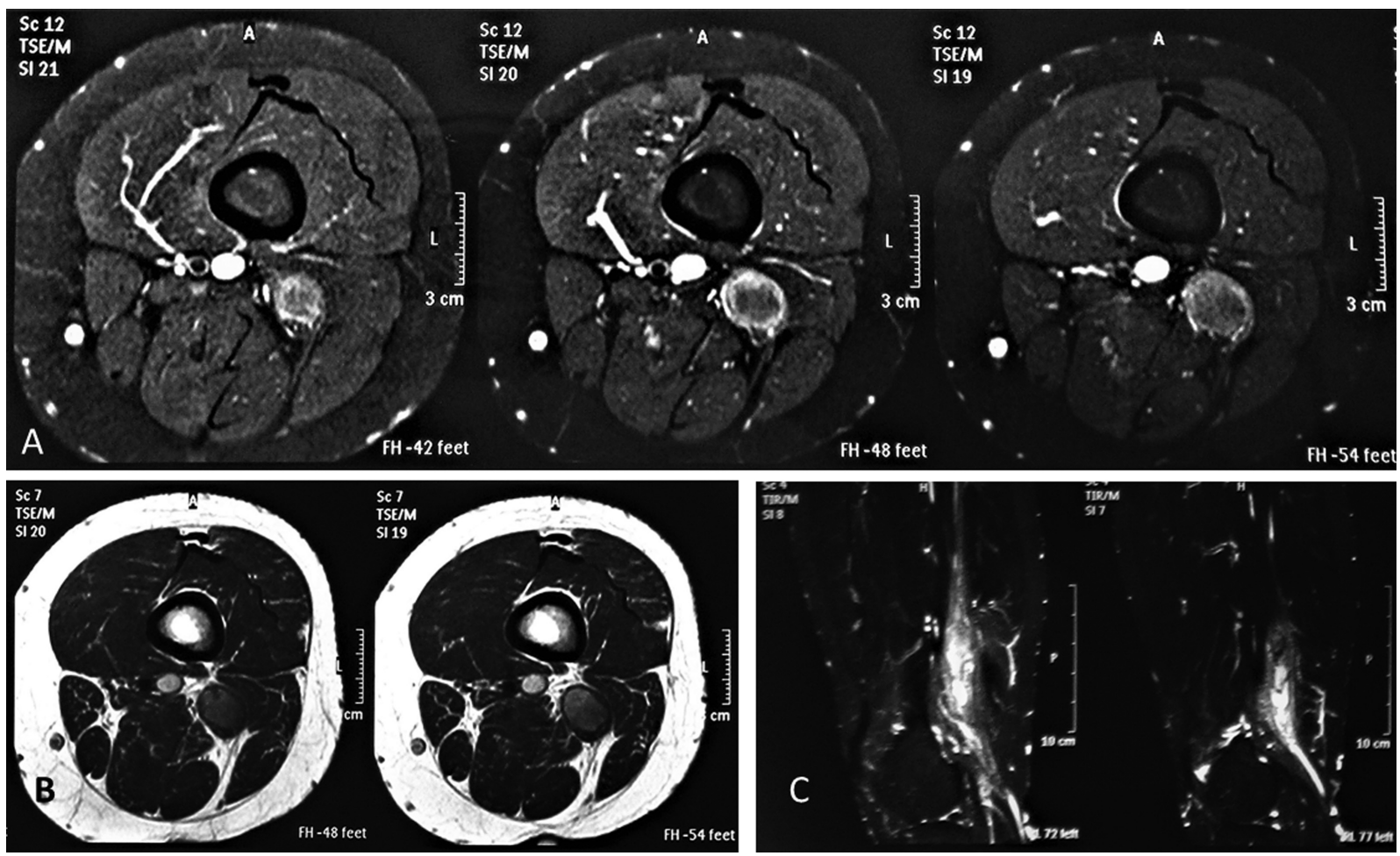

Fig. 1 (A) T1-weighted magnetic resonance imaging (MRI) in axial sections showing uptake of the contrast, more intense at the periphery.

(B) T1-weighted MRI in axial sections showing regular lesion of well-defined limits without invasion of neighboring planes and without surrounding edema.

(C) T2-weighted short tau inversion recovery (STIR) MRI in sagittal sections showing sciatic nerve damage in the topography of its bifurcation. 


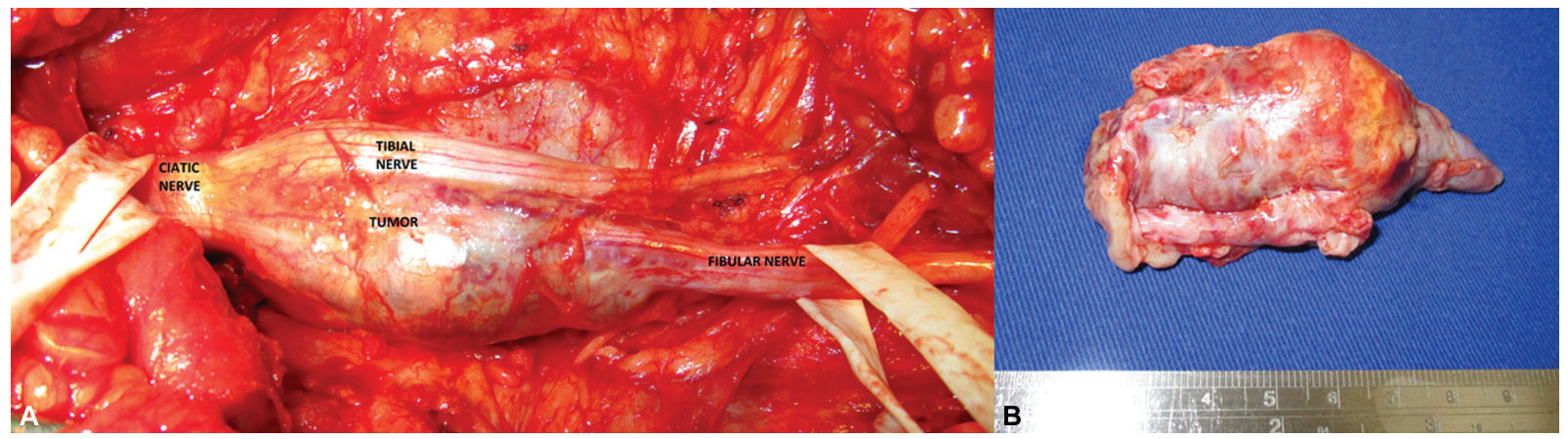

Fig. 2 (A) Photograph of the surgical field showing a tumor on the left sciatic nerve, affecting its circumscribed fibular portion, with no signs of invasion of neighboring tissues. (B) Photograph of the 7-cm long surgical specimen.

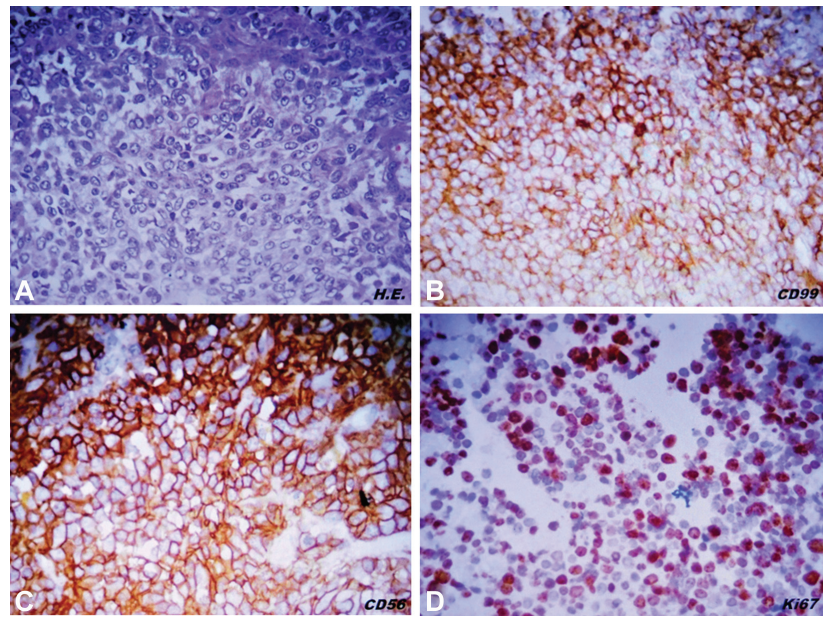

Fig. 3 (A) Slide stained with hematoxylin and eosin showing a small, blue, regular cell tumor with large nuclei and fine chromatin; magnification: 20X. (B) CD99 positivity on immunohistochemistry; magnification: 20X. (C) Positivity for CD56 on immunohistochemistry; magnification: 20X. (D) Positivity for $\mathrm{K}_{\mathrm{i}} 67$ in $70 \%$ of tumor cells; magnification: $20 \mathrm{X}$

signs of neurological recovery started with 10 months of the surgery, and progressed until the 14th postoperative month, after which it stopped, despite the physical therapy treatment for 24 months after the surgery. In the first postoperative year, the patient underwent local radiological control with MRI scans every 3 months and chest tomography every 6 months; in the second postoperative year, the patient underwent local radiological control with MRI scans every 6 months. The last local control with MRI was performed at the 28th postoperative month, without evidence of local recurrence ( - Fig. 4). The patient is still being followed-up by oncology.

\section{Discussion}

Ewing sarcoma (ES) is a rare malignancy that affects bones and soft tissues. Its incidence was of 2.93 per 1 million inhabitants in the United States between 1973 and 2004. ${ }^{2}$ Despite this, it is the second most common malignant bone tumor in children and adolescents. The peak incidence occurs at 10 and 20 years of age, being uncommon after 30 years of age. Ewing sarcoma has a predilection for the male sex (male/female ratio: $1.3-1.5: 1$ ), it is more frequent in Caucasians than in Asians and Africans or African-Americans. ${ }^{4}$ Currently, ES is grouped with other tumors that share the same histological and genotypic characteristics, forming ESFT, which includes ES of bone, extraosseous ES (peripheral neuroepithelioma), Askin tumor, and peripheral PNET. ${ }^{5}$ From the histological point of view, the ESFT presents as small, round, blue cell tumors when stained with hematoxylin and eosin (H\&E), homogeneous, containing varying amounts of necrosis. Additional findings of rosettes and pseudorosettes, indicating neuronal differentiation, may also be observed in cases of PNET. ${ }^{6}$ Another characteristic common to ESFT is the $\mathrm{t}(11 ; 22)(\mathrm{q} 24 ; \mathrm{q} 12)$ translocation

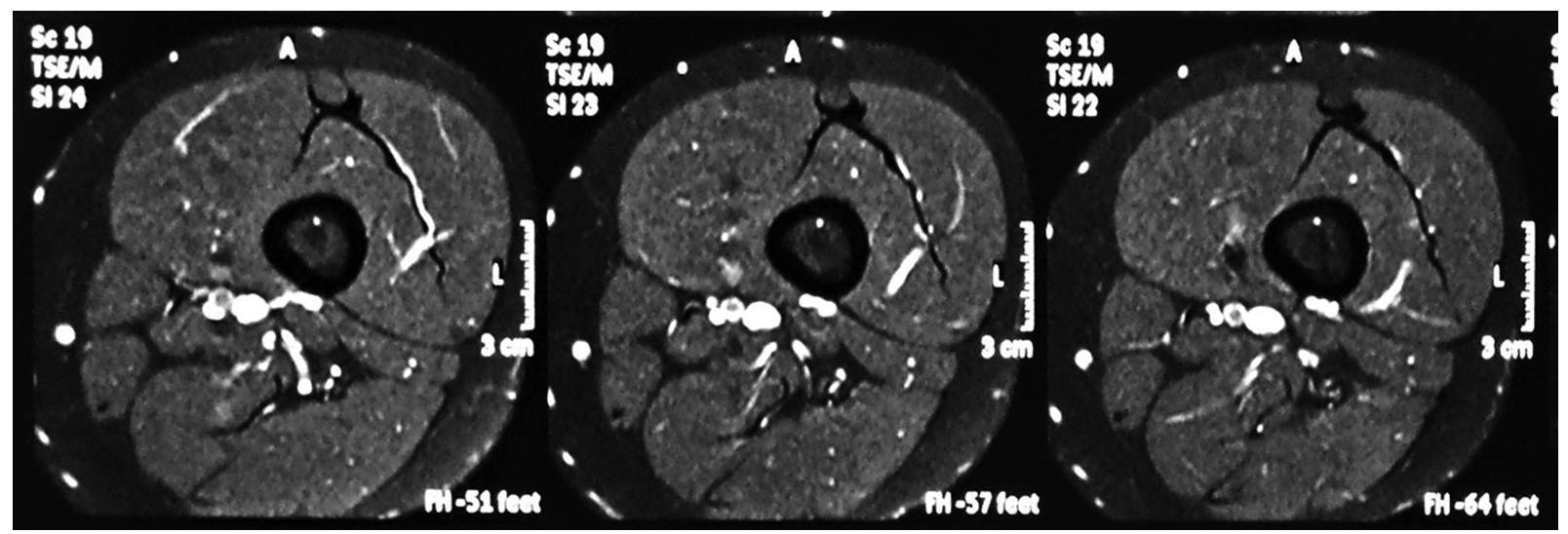

Fig. 4 T1-weighted contrast-enhanced MRI 28 months after the surgical treatment, with no signs of local recurrence. 
that produces a chimeric gene called EWSR1-FLI1, which occurs in $\sim 85 \%$ of tumors. Another fusion, which occurs in $10 \%$ of cases, is the result of translocation $\mathrm{t}$ (21:22) (q22; q12), which generates the EWSR1-ERG gene. These fusions encode chimeric proteins that function as aberrant oncogenic factors. On immunohistochemistry, about 90\% of ESFT cases are positive for CD99, a membrane glycoprotein product of the MIC2 gene. ${ }^{5-7}$ There are hypotheses about the origin of these tumors, among them: the theory of parental mesenchymal stem cells, and that of an origin in a primitive nerve cell or in the postganglionic cholinergic neurons of the parasympathetic system; however, the true progenitor cell of these tumors remains unknown. ${ }^{1,3,7}$

About 25\% of cases of ESFT are in soft tissues, affecting patients between 10 and 30 years of age, with a peak incidence at 20 years of age. The most frequent sites include the chest wall, the paravertebral musculature, the lower extremities, and the retroperitoneal space. ${ }^{4}$ Head and neck involvement is uncommon. $^{8}$ There are reports of renal $^{9}$ and pancreatic ES, ${ }^{10}$ and cases of ESFT originating in nerves are very rare. It is interesting to note that probably the first report of ESFT was made by Stout et al. in 1918, who described a case of tumor originating in the ulnar nerve, composed of round and undifferentiated cells that exhibited the formation of rosettes, and that the concept of neuroepithelioma was later proposed. ${ }^{11}$ Reports of other cases of ESFT originating in the nerves are scarce. Nesbitt and Vidone ${ }^{12}$ reported a case of PNET of the sciatic nerve in a 6-year-old patient; Martins et al. ${ }^{13}$ reported a case of PNET arising from a branch of the sciatic nerve in a 17year-old female patient; and Akeyson et al. ${ }^{14}$ described a case of PNET originating in the median nerve of an elderly patient. They are typically fast-growing tumors, usually painful. Approximately $1 / 3$ of the cases of extraosseous ES have distant metastases at the time of the initial diagnosis, but extraskeletal ES is considered a systemic lesion since its presentation, even without documented metastasis. The initial assessment is performed with MRI scans of the affected site followed by a biopsy of the tumor with a needle or an open procedure. Staging is performed with chest tomography and whole-body scintigraphy. The performance of bone marrow biopsy in the initial stage is controversial. Among the factors with the worst prognosis, the existence of metastasis in the initial evaluation is the most significant. Patients with a single lung metastasis have a slightly more favorable prognosis than those with metastases in other regions. Other unfavorable prognostic factors include age $>10$ years, tumor larger than $8 \mathrm{~cm}$ or with volume $>200 \mathrm{~mL}$, central lesions (in the pelvis or spine), and low response to chemotherapy. ${ }^{15}$

The treatment consists of systemic chemotherapy associated with local treatment (surgery and/or radiotherapy). The drugs proven effective for the treatment of ESFT include doxorubicin, cyclophosphamide, vincristine, actinomycin-D, ifosfamide, and etoposide. Currently, the regimen with VDCIE is the protocol used in countries in North America and in many countries in Europe. The response to chemotherapy is an important prognostic factor. For local control, radical surgical resection is the best option. For those cases in which the lesion cannot be completely removed or in the absence of an adequate safety margin, local radiotherapy would be indicated. $2,4,6,11,15$

Considering the aforementioned information, the ideal case, the one with the best prognosis, is the patient with a localized tumor, who responds adequately to chemotherapy, and its complete surgical resection with a safety margin is possible. This raises many questions in relation to the case herein described. First, the initial diagnostic hypothesis of an ESFT, due to the rarity of the pathology, would have never been considered a probable option. There are no elements in the clinical history and neurological examination that can differentiate it from other more frequent peripheral nerve tumors such as schwannomas and neurofibromas. Second, the subsidiary exams did not provide elements that would suggest the diagnosis of this illness. The anatomical MRI exam was accurate in relation to the tumor topography and its limits, but insufficient to indicate the histological type and detect signs of malignancy. In a study comparing the characteristics of neurofibromas and malignant tumors of nerve sheaths on MRI, Wasa et al. ${ }^{16}$ described some aspects that could facilitate differentiation: mass dimensions, since malignant lesions tend to be larger; the perilesional enhancement; the perilesional edema zone; and the intralesional cystic lesion. The presence of two or more of these characteristics suggested a malignant tumor of the nerve sheath with a sensitivity of $61 \%$ and specificity of $90 \%$. There are no studies comparing the aspects of nerve ESFT MRI with those of other tumors. Using the data found by Wasa et al. ${ }^{16}$ in our case, we would not find any indication of malignancy, because the tumor dimensions were not bulky, and there was no perilesional edema or intralesional cystic lesion. The observed peripheral enhancement can be found in neurofibromas and even in schwannomas. ${ }^{17,18}$ Nerve tractography and functional MRI can be valuable tools to differentiate between benign and malignant tumors of the peripheral nerves; however more studies are needed to elucidate this question. ${ }^{19-21}$

Another aspect to be discussed is the surgical treatment performed. Most peripheral nerve tumors are composed of benign lesions, and the incidence of malignant peripheral nerve tumors is of around $0.001 \%$. In a brief review, Ball and Biggs $^{22}$ described the steps of the surgical management of benign peripheral nerve tumors (BPNTs). Clinical history and propedeutic with appropriate electrophysiological and imaging tests are essential. Percutaneous biopsies of nerve tumors should be avoided, as there is a risk of nerve damage, of spread of tumor cells through the needle path, and of formation of scars that can hinder complete lesion resection. The general principles of BPNT surgical resection include adequate exposure of the lesion, including the healthy proximal and distal portions of the affected nerve, tumor inspection and intraneural dissection to identify and separate uninvolved fascicles, and electrical stimulation to identify functional fascicles. The treatment protocol for ESFT advises to initially perform a tumor biopsy to confirm the diagnosis, followed by neoadjuvant chemotherapy to observe the response. After these steps, local treatment with radical surgery with a safety margin and/or radiation therapy are indicated. In the case herein reported, we changed the order recommended in the treatment protocol for ESFT: the 
surgical treatment preceded the other treatments, and this was due to a failure in the preoperative diagnosis. How this will affect the patients prognosis remains to be seen. Another aspect of the surgical treatment to be discussed is the reconstruction of the affected fascicles with a sural nerve graft. Had the diagnosis of ESFT been known, reconstruction with a graft at this time might have been contraindicated because there would be a need for radical resection with a safety margin, although there are no data in the literature to specifically guide this situation. On the other hand, it is interesting to observe the neurological evolution of this patient. Historically, the functional prognosis of patients with traumatic sciatic nerve injuries in the fibular portion or lesions in the common fibular nerve that require graft reconstruction is not favorable, especially in those cases in which the use of long grafts was necessary. ${ }^{23,24} \mathrm{Kim}$ et al. ${ }^{25}$ evaluated the result of the surgical treatment of traumatic fibular nerve injuries and observed that, in those in which reconstruction with a graft larger than $6 \mathrm{~cm}$ was necessary, a good functional result occurred in only $44 \%$ of cases. In the case herein reported, in addition to the use of grafts for reconstruction, there was a need for local control with radiotherapy, as tumor resection was not performed with a safety margin, and exposure to prolonged chemotherapy with multiple drugs, especially vincristine, which is known to cause sensory-motor neuropathy. The effects of radiation on the peripheral nerves and the possibility of postirradiation neuropathy are well known, ${ }^{26,27}$ but there is insufficient data regarding the influence of radiotherapy on nerves reconstructed with a graft. Even in this context, the patient described presented some degree of neurological recovery, especially sensitivity, indicating that there is possibility of nerve regeneration. Therefore, the case herein described offers the possibility of discussing several issues regarding malignant tumors of peripheral nerves, such as the difficulty in establishing the preoperative diagnosis, the limitation of imaging tests to differentiate benign from malignant lesions, and, therefore, the adequate surgical management of these injuries.

\section{Conflict of Interests}

The authors have no conflict of interests to declare.

\section{References}

1 Croci AT, Camargo OP, Oliveira NRB. Tratamento cirúrgico do sarcoma de Ewing: avaliação oncológica e funcional. Rev Bras Ortop 1996;31:909-918

2 Balamuth NJ, Womer RB. Ewing's sarcoma. Lancet Oncol 2010;11 (02):184-192

3 Pereira RJS, Araújo R, Araújo SCP, et al. Sarcoma de Ewing extraósseo. Arquivos Brasileiros de Ciências da saúde 2010;35:65-68

4 Iwamoto Y. Diagnosis and treatment of Ewing's sarcoma. Jpn J Clin Oncol 2007;37(02):79-89

5 Zagar TM, Triche TJ, Kinsella TJ. Extraosseous Ewing's sarcoma: 25 years later. J Clin Oncol 2008;26(26):4230-4232

6 Davis LE, Malempati S. Ewing sarcoma in adolescentes and Young adults:diagnosis and treatment. Clin Oncol Adolesc Young Adults 2014;4:21-31
7 Rani PSV, Shyamala K, Girish HC, Murgod S. Pathogenesis of Ewing sarcoma: A review. Journal of Advanced Clinical \& Research Insights 2015;2:164-168

8 Martínez NV, Rosas DC, Alba LM, et al. Sarcoma de Ewing extraósseo em cabeza y cuello:revisión de la literatura a propósito de um caso. Res Esp Cir Oral Maxilofac 2017;39(02):105-112

9 Alonso AH, Gárate MM, Amo FH, et al. Sarcoma de Ewing renal primário. Arch Esp Urol 2011;64(07):636-639

10 Komforti MK, Sokolovskaya E, D’Agostino CA, Benayed R, Thomas RM. Extra-osseous Ewing sarcoma of the pancreas: case report with radiologic, pathologic, and molecular correlation, and brief review of the literature. Virchows Arch 2018;473(03):361-369

11 Ozaki T. Diagnosis and treatment of Ewing sarcoma of the bone: a review article. J Orthop Sci 2015;20(02):250-263

12 Nesbitt KA, Vidone RA. Primitive neuroectodermal tumor (neuroblastoma) arising in sciatic nerve of a child. Cancer 1976;37 (03):1562-1570

13 Martins RS, Siqueira MG, Moraes OJS, et al. Tumor neuroectodérmico primitivo periférico originado em ramo do nervo ciático. Braz Neurosurg 2002;21(3-4):116-119

14 Akeyson EW, McCutcheon IE, Pershouse MA, Steck PA, Fuller GN. Primitive neuroectodermal tumor of the median nerve. Case report with cytogenetic analysis. J Neurosurg 1996;85(01):163-169

15 Gaspar N, Hawkins DS, Dirksen U, et al. Ewing Sarcoma: Current Management and Future Approaches Through Collaboration. J Clin Oncol 2015;33(27):3036-3046

16 Wasa J, Nishida Y, Tsukushi S, et al. MRI features in the differentiation of malignant peripheral nerve sheath tumors and neurofibromas. AJR Am J Roentgenol 2010;194(06):1568-1574

17 Lee SK, Kim JY, Lee YS, Jeong HS. Intramuscular peripheral nerve sheath tumors: schwannoma, ancient schwannoma, and neurofibroma. Skeletal Radiol 2020;49(06):967-975

18 Bhargava R, Parham DM, Lasater OE, Chari RS, Chen G, Fletcher BD. MR imaging differentiation of benign and malignant peripheral nerve sheath tumors: use of the target sign. Pediatr Radiol 1997; 27(02):124-129

19 Filler AG, Kliot M, Howe FA, et al. Application of magnetic resonance neurography in the evaluation of patients with peripheral nerve pathology. J Neurosurg 1996;85(02):299-309

20 Filler A. Magnetic resonance neurography and diffusion tensor imaging: origins, history, and clinical impact of the first 50,000 cases with an assessment of efficacy and utility in a prospective 5000-patient study group. Neurosurgery 2009;65(4, Suppl) A29-A43

21 Chhabra A, Thakkar RS, Andreisek G, et al. Anatomic MR imaging and functional diffusion tensor imaging of peripheral nerve tumors and tumorlike conditions. AJNR Am J Neuroradiol 2013; 34(04):802-807

22 Ball JR, Biggs MT. Operative steps in management of benign nerve sheath tumors. Neurosurg Focus 2007;22(06):E7

23 Samardzić MM, Rasulić LG, Grujicić DM. Results of cable graft technique in repair of large nerve trunk lesions. Acta Neurochir (Wien) 1998;140(11):1177-1182

24 Roganovic Z. Missile-caused complete lesions of the peroneal nerve and peroneal division of the sciatic nerve: results of 157 repairs. Neurosurgery 2005;57(06):1201-1212, discussion 1201-1212

25 Kim DH, Murovic JA, Tiel RL, Kline DG. Management and outcomes in 318 operative common peroneal nerve lesions at the Louisiana State University Health Sciences Center. Neurosurgery 2004;54 (06):1421-1428, discussion 1428-1429

26 Delanian S, Lefaix JL, Pradat PF. Radiation-induced neuropathy in cancer survivors. Radiother Oncol 2012;105(03):273-282

27 Gikas PD, Hanna SA, Aston W, et al. Post-radiation sciatic neuropathy: a case report and review of the literature. World J Surg Oncol 2008;6(06):130 